\title{
Behavioral Patterns of Tobacco Addiction of Students at Some Universities in HCMC - Viet Nam
}

\author{
Huynh Van Son \\ HCMC University of Education \\ Vietnam \\ E-mail: sonhuynhts@gmail.com \\ Mai My Hanh \\ HCMC University of Education \\ Vietnam \\ E-mail: hanhmai1012@gmail.com \\ Nguyen Thi Diem My \\ HCMC University of Education \\ Vietnam \\ E-mail: diemmytlgd@gmail.com
}

Received: March 20, 2014 Accepted: April 11, 2014 Published: June 8, 2014

doi:10.5296/ijch.v1i1.5709

URL: http://dx.doi.org/10.5296/ijch.v1i1.5709

\begin{abstract}
Article refers to behavioral patterns of tobacco addiction of students at some universities in Ho Chi Minh City, Vietnam. The study results showed that $76.85 \%$ of students have non-smoking and $20.0 \%$ of students currently smoke. For $20 \%$ above, there is $27.6 \%$ of the students have mild addiction, $26.7 \%$ of students have moderate addiction and $22.9 \%$ of students have trend addiction. This really is a concerning figure for tobacco addiction behavior in particular and cultural life of Vietnam in general.
\end{abstract}

Keywords: Behavior, Behavior of tobacco addiction, Behavioral patterns of tobacco addiction 


\section{Introduction}

In the past ten years, a series of new problems related to deviant behavior in human psychology have been applied and researched in a variety of different angles. One of those issues is the study of addiction behavior. A series of addiction behavior problems in modern life were researched on theoretical aspects as well as in clinical practice, such as Internet addiction, shopping addiction, sex addiction, drug addiction, cigarette addiction,etc. In the studies of the addiction behavior problems related to substance use causing psychological dependence, the consequences on the level of mental and health, personal and social levels are concerned by the society. One of addiction behaviors related to drugs are always interested to overcome by the society is tobacco addiction behavior (VINACOS, 2013)

According to the Ministry of Health ( $\mathrm{MOH})$, currently, there is about $50 \%$ of adult males smoke in our country (approximately 17 million smokers). With this figure, Vietnam has become the nation with the highest smoking rate in the world. Tobacco not only causes serious diseases, even cancer for people who smoke directly but also at higher risk for those around him. About 40,000 of Vietnamese people die from tobacco every year. Smoking is the cause of 25 dangerous diseases to humans. If there is no preventive measures, by 2020 , the country will have $10 \%$ of the population die from diseases related to use of tobacco products (WHO, 2008).

The most notably in the figure above is the highest smoking rate is at universities, nearly $55 \%$. Without timely measures to prevent, smoking behavior will lead serious consequences. According to author S. Griffiths, addiction behavior is understood as the dependence of individuals involved in some specific activities, despite harmful consequences, such as the decline of health, mental and social life. Therefore, understanding particularly on the status of smoking behavior in general and in particular and tobacco addiction of some students at universities plays an essential role. (VINACOS, 2013)

\section{Research Method}

Topic is the combination of many research methods, including the questionnaire survey is the main method, the left methods are complementary ones. Survey Questionnaire Method: The main questionnaire consists of two questionnaires for students after choosing carefully and for medical doctors, psychiatrists, psychologist therapists. The contents of the survey contain 11 questions to find out the current status of tobacco addiction behaviors in students. From number1 to number11 each question is counted scores and will be figured out total scores to determine the tobacco addiction level. The questions have 5 levels are figured out as follows: 
Table 1 . The way to count into score each question in the official questionnaire

\begin{tabular}{|c|c|}
\hline \multirow{2}{*}{ Average } & Levels \\
\cline { 2 - 2 } & Number $\mathbf{3 , 4 , 5 , 6 , 7 , 8 , 9 , 1 0}$ \\
\hline $3.51-4$ & Very Regularly \\
\hline $2.51-3.5$ & Regularly \\
\hline $1.51-2.5$ & Sometimes \\
\hline $0.5-1.5$ & Seldom \\
\hline $0-0.49$ & Never \\
\hline
\end{tabular}

Except for number 1, 2 and 11, respectively grading from 1 to 4 scores for answers from the time level and amount of smoking from low to high, the behavior from inappropriate to the most appropriate.

Based on the lowest and highest total scores, tobacco addiction levels will be figured out as follows:

Table 2. The way to count tobacco addiction level

\begin{tabular}{|c|c|}
\hline Scores & Tobacco Addiction Level \\
\hline $281-360$ & Serious Addiction \\
\hline $211-280$ & Addiction \\
\hline $141-210$ & Light Addiction \\
\hline $71-140$ & Tend to Abuse Tobacco \\
\hline $4-70$ & Use in Usual Way \\
\hline
\end{tabular}

Second, the questionnaire for the medical doctors, practicing psychologist also structured like questionnaire for students

\section{Results and Discussion}

\subsection{Smoking Behavior Status of Students}

To find out smoking behavior status of students, we surveyed 700 students at seven universities in Ho Chi Minh city. Of 700 students in the survey, there were 538 students confirmed that they do not smoke, corresponding to the rate of $76.85 \%$ and second position which is the number of student who smoke, relative to 140 students corresponding to $20.0 \%$, of which 132 students are male (18.85\%) and 8 females $(1.15 \%)$. 
Table 3. Smoking behavior status of students

\begin{tabular}{|c|l|r|r|}
\hline Number & \multicolumn{1}{|c|}{ CONTENT } & Frequency & Rate (\%) \\
\hline 1 & Non-smoking & 538 & 76.85 \\
\hline 2 & Smoke & 140 & 20.00 \\
\hline 3 & Will smoke & 12 & 1.71 \\
\hline 4 & Used to smoke & 10 & 1,42 \\
\hline
\end{tabular}

The number of $20 \%$ of students currently smoke is much lower compared with statistical data that WTO surveyed the smoking rate of population of Vietnam in 2010. According to the World Health Organization (WHO), Vietnam is considered as one of the countries with the highest smoking rates in the world with $56.1 \%$ in men and $1.8 \%$ in women (WHO, 2008).

According to Global Adults on Tobacco Smoking in (GATS) conducted in Vietnam in 2010, Vietnam had the percentage of men using tobacco very high compared to other countries in the world and in the region with $47.4 \%$ of adult men and women smokers are $1.4 \%$ and $23.8 \%$ of adults in general (15.3 million adults) are smokers. Thus $23.8 \%$ of adults have been smoked or will smoke is quite corresponding to data collected from th topic . Comparing the results of students are smoking with the research of Do Van Dung (researched in 2002) shows the rate of smoking in men is $14.25 \%$ and $0.08 \%$ in women with total number is nearly $14: 33 \%$. But this is figure conducted in 2002 and with lots of people, so it is difficult to compare with the most appropriate way .However, the ratio between male and female smokers is quite suitable. More specifically, the number of students who are smokers is $20 \%$, higher than the figures of Do Van Dung is quite consistent with predictions of the Vietnam Ministry of Health that smoking in Vietnam will tend to increase and rejuvenate for 2030 with the ongoing reality. (Do, 2003)

In summary, the initial collective data with $140(20 \%)$ of students smoking is a highly reliable figure when compared with those of other studies, prestigious organizations in the world. We can explain $20 \%$ of Vietnamese students are smoking by the following reasons:

- According to the Vietnamese customs, "The betel starts story", it will be lack when mentioning betel without pipe tobacco. Most women chew betel, and for the men, pipe tobacco goes with them when happy, sad even their lifetime. Pipe tobacco is smoked in pipes, pipe bowls ... for convenience when being away from home, should smoke by plow pipe (pipe for smoking while plowing in the fields so-called plow pipe). The smoking pipe habit is "prelude" in the reunion, soy puff, can be considered a culture of social village class in the feudal. Smoking for fun, for meeting, after time working hard ...for relieving hunger, "smoking pipe talking about the world", for reducing addiction... because of these, it was raised "smoking pipe tobacco culture". In Vietnamese rural, before most of family always smoked pipe, when they smoke it drives them tipsy, and then enraptured. Today, pipe tobacco has been replaced with a cigarette and become an indispensable habbit.

- Teenagers smoke by imitating adults and as a way to prove themselves no more to be children. In the family, if parents are addicted to smoke, their children are easy to addict. This 
is a problem related to culture, tradition, each family's lifestyle.

- The Vietnamese or smoking invitation habbit though people are invited to smoke or not. Taking and litting a cigarette as a "polite" act. This habit has been passed from generation to generation. This increased sense of confidence when contacting, talking and communicating with people around.

- Tobacco is also suited to pockets of Vietnam people, especially students. The tobacco factories appear everywhere. Finally propaganda issues cigarettes is still not really popular in Vietnam.

However, 140 objects are just initial collective ones. In order to more accurately determine the proportion of students who smoke to collect the objects for the study, the researchers used the table to learn more about smoking cessation status of the student to control data. Thereby indirectly assess the reliability of the original figure of $20 \%$ of students who are smokers.

The data in Table 2 shows, the number of students who do not give up smoking is 100 students, corresponding to $14.28 \%$ and 40 is the number of students who try to give up tobacco, but failed, corresponding to $5.71 \%$. Thus, the total of these two groups corresponding to $20 \%$ of students who are smokers. Similarly, the number of students who can give up smoking successfully is $10(1.42 \%)$ and do not smoke is $550(78.57 \%)$. Totally, two groups are corresponding to group of students who have ever used is 10 students, do not smoke is 358 students and will smoke is 10 students.

Table 4. Status of tobacco give-up of students

\begin{tabular}{|c|l|l|l|}
\hline Number & \multicolumn{1}{|c|}{ CONTENT } & \multicolumn{1}{c|}{ Frequency } & \multicolumn{1}{c|}{ Rate (\%) } \\
\hline 1 & No use of tobacco & 550 & 78.57 \\
\hline 2 & No try to give up & 100 & 14.28 \\
\hline 3 & Try to give up without success & 40 & 5.71 \\
\hline 4 & Give up with success & 10 & 1.42 \\
\hline
\end{tabular}

\subsection{Level of Tobacco Addiction of Students}

Table 5. Level of tobacco addiction of students

\begin{tabular}{|c|l|c|c|}
\hline No. & \multicolumn{1}{|c|}{ LEVEL } & Frequency & Rate (\%) \\
\hline 1 & Mild addiction & 29 & 27.6 \\
\hline 2 & Average addiction & 28 & 26.7 \\
\hline 3 & Trend of tobacco abuse & 24 & 22.9 \\
\hline 4 & Nornal use of tobacco & 16 & 15.2 \\
\hline 5 & Heavy addiction & 8 & 7.6 \\
\hline
\end{tabular}


Of 105 students were selected from the screening questionnaire, the majority of students are concentrated in two levels, it is mild addiction and average addiction. Of these, 29 students $(27.6 \%)$ fall into mild addiction level, accounting for nearly one third objective smokers. At this level of addiction, individuals are dependent on tobacco, they also appear unpleasant behaviors when they cannot smoke, avoid public places, responsibility for smoking. However, smoking behavior and the expression of negative behavior when they use drugs are still in control when there is an effort in terms of will and a reminder of the people around. The study results from the case of NMD shows: "I also smoke regularly during the day, and the average of two or more packages. But when I need time for the job or do not want to bother my friends and relatives for the smoke, I can restrain myself and smoke later or at another place."

The next level is average addiction with 28 students $(26.7 \%)$. This figure suggests that when students have the habit of smoking regularly for some time and a certain intensity, they can easily be addicted. The level of addiction increases over time and smoking intensity. At this stage, students tend to smoke regularly. They avoid the prohibition of smoking in public places to perform smoking behavior, such as walking away, temporarily stopping working. They can be aware of their negative behaviors but cannot stop and start accepting probable consequences to themselves and others. Case study from object TMB, we found the following results: "Every day I smoke two packs of cigarettes and often smoke continuously when I feel tired or stressed. I think I cannot give up."

Behind mild addiction and average is the trend of tobacco abuse with 24 students $(22.9 \%)$, this level also accounts for nearly one fifth of the sample. Although this trend is not in the level of tobacco addiction but it still has some dominant behaviors in their personal lives. The density of tobacco use is more frequent in nature and this behavior begins to dominate life and their activities. However, they can still control their smoking behavior in different situations in an appropriate manner.

Behind those three levels, using tobacco normally is 16 people $(15.2 \%)$, under one fifth of the objects in the study. This figures is really concerning because it demonstrates the problems that tobacco use will have fewer individuals using tobacco normally but there is a risk tends to fall into tobacco abuse and the tobacco addiction. At the stage of using tobacco normally, the users are not totally dependent. They use tobacco as a behavior of social courtesy, improvisational nature, there is no psychological coercion. When using tobacco with this nature, they can actively control the situation of smoking.

Finally, of 105 students whose smoking behavior, there are only 8 students at a rate of $7.6 \%$ fall in the level of serious addiction. Although it is under $1 / 10$ of the objects in the study, but the level of addiction and severe manifestations and consequences of disturbing the $7.6 \%$ figure is also considerable numbers. At this level, despite the personal efforts in terms of willpower but they still cannot stop using tobacco with regular level, often accompanied by the expression of negative behaviors such as irritability if not smoke, absolutely cannot concentrate or work in the absence of tobacco, tobacco use is prohibiting place, still perform smoking behavior without caring request and remind of other people around, etc. In short, 
dependence on cigarette makes individual cannot control themselves and despite all the negative behavior as well as consequences of smoking to satisfy the use of tobacco.

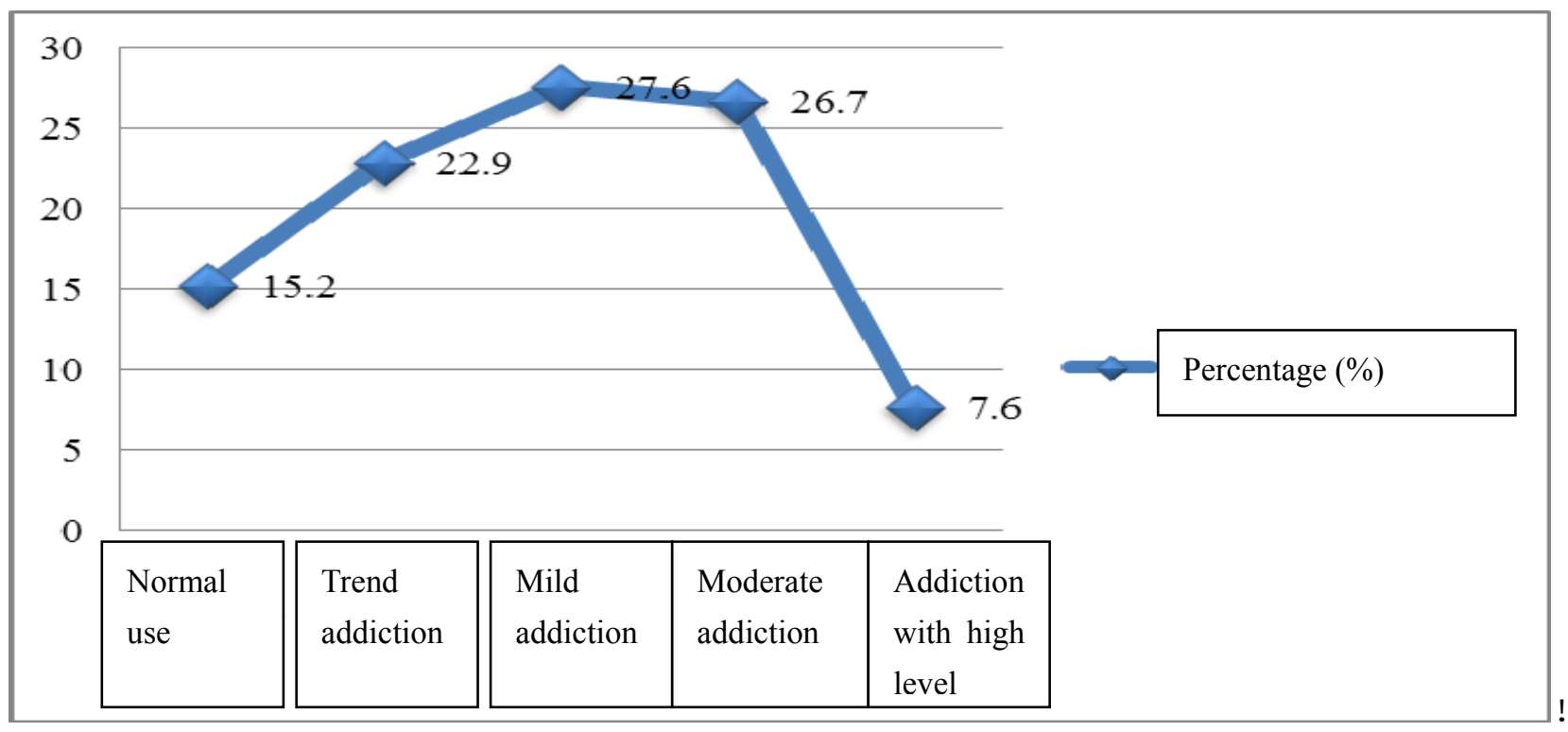

Figure 1. Tobacco Addiction of students

The study results also quite similar to some studies about cigarette addiction level of some researchers in Medicine, Sociology. All researches of those authors use brief Test Fagerstrom and Test Hom to study the tobacco addiction degree of objectivity of tobacco addiction. It can be seen that the degree of tobacco addiction of students is lower than the group that other researchers have examined. However, assessment of general aspects, we can find that the proportion of tobacco addiction of the objectivity groups - including students is at a rate of over $60 \%$. This confirms more strongly that when a person smokes, that individual is very easy to be addicted. Figures compared also showed with a higher age, the longer duration of exposure to tobacco, the higher increase in addiction tend. Therefore, interventions and smoking cessation measures should be conducted as soon as possible so that the prevention of the harmful effects of tobacco can be really effective (Le, 2007; Lam, Tse, Yu \& Griffiths, 2009)

\section{Conclusion}

Students are the core force plays an important role in contribution of society. Mental and physical strength have the significance in the "strength" of society. Tobacco addiction behavior of students stated above is actually a figure to pay attention, especially when considering the cultural life of Vietnamese people. On 18/6/2012, Congress enacted the Law on Prevention of the harmful effects of tobacco, including the provisions of Article 9 prohibits people under 18 years old to use , buy, sell tobacco; supply tobacco for people under 18 years old, etc. But the implementation has not been implemented strongly yet, especially, the sale of tobacco around universities, colleges and high schools still takes place.

In that reality, the activities of prevention and anti-smoking is an urgent problem. Moreover, the object is most interested is the youth, with the typical age mentality is very easy to be 


\section{Macrothink}

International Journal of Culture and History

ISSN 2332-5518 2014, Vol. 1, No. 1

drawn into smoking. Therefore, the prevention of smoking behavior at the age of students in Vietnam is very important because the rate of population structure at this age is quite high and this is the age most susceptible and they are not aware of $t$ the harmful effects of tobacco.

\section{References}

Do V. D. (2003). Smoking rate of high school students, university students, in the Southern in 2002. Medical Magazine in 2002, 7(1).

Lam, T. S., Tse, L. A., Yu, I. T., \& Griffiths, S. (2009, January). Prevalence of smoking and environmental tobacco smoke exposure, and attitudes and beliefs towards tobacco control among Hong Kong medical students. Public Health. http://dx.doi.org/10.1016/j.puhe.2008.07.016

Le, K. B. (2007). Survey on smoking of third-year students at Ho Chi Minh city University of Medicine TP.HCM. Medical Magazine of Ho Chi Minh city, 11(1).

Program of tobacco affect prevention (VINACOS). (2013). Guide book for give up smoking.

World Health Organization, Geneva, WHO. (2008). Report on the global to bacon epidemic (from WHO). The M POWER package.

\section{Copyright Disclaimer}

Copyright reserved by the author(s).

This article is an open-access article distributed under the terms and conditions of the Creative Commons Attribution license (http://creativecommons.org/licenses/by/3.0/). 